\title{
Extraventricular Neurocytoma
}

National Cancer Institute

\section{Source}

National Cancer Institute. Extraventricular Neurocytoma. NCI Thesaurus. Code C92555.

A WHO grade II neoplasm that arises from the brain parenchyma. Morphologically it is characterized by the presence of neoplastic uniform, round cells with neuronal differentiation. Unlike central neurocytoma, it does not involve the lateral ventricles. It usually affects young adults and has a favorable prognosis. 